\title{
Number of Early Term Births
}

National Cancer Institute

\section{Source}

National Cancer Institute. Number of Early Term Births. NCI Thesaurus. Code C122189.

A measurement of the total number of birth events at which the gestational age of the neonate is 37 weeks and 0 days through 38 weeks and 6 days. 